\title{
Therapeutic Considerations in Individuals at Clinical Risk for Developing Psychosis
}

Fiza Singh, $M D^{1,2^{*}}$

Meriah DeJoseph, $B S^{2}$

Kristin S. Cadenhead, $M D^{1,2}$

\author{
Address \\ ${ }^{1}$ San Diego Veterans Administration Healthcare System (SDVAHS), La Jolla, CA, USA \\ $2,{ }^{\star}$ Department of Psychiatry, University of California at San Diego (UCSD), 0810, \\ 9500 Gilman Drive, La Jolla, CA 92093-0810, USA \\ E-mail: fsingh@ucsd.edu
}

Published online: 14 March 2014

(C) Springer International Publishing AG 2014

Keywords Clinical high risk (CHR) - Psychosis - Staging - Diet - Lifestyle - Antipsychotics - Antidepressants . Psychotherapy - Omega-3 fatty acids - Oxytocin - Minocycline - Glutamate modulators - Cognitive training . Neurofeedback

\section{Opinion statement}

In the last two decades, increased focus has been placed on earlier identification and treatment of psychotic illness in an effort to prevent illness onset and/or long-term sequelae of schizophrenia. Typically, a 16- or 17 -year-old young man or woman is referred to our clinic by a mental health provider who has noted thought disorder (referential thoughts, suspiciousness) in addition to depression and/or anxiety. The individual is usually someone who has stopped socializing with friends, has poor grades, has experimented with illicit substances (frequently cannabis), endorses unusual beliefs, but maintains insight. We begin by obtaining a thorough history, including collateral sources such as family and school counselors and a medical history to rule out any organic causes. Oftentimes there is a family history of schizophrenia or bipolar disorder, traumatic childhood, and at times a history of social discomfort and bullying. It is not uncommon for our clients to endorse depression and social anxiety in addition to prepsychotic perceptual abnormalities. In our clinic, we use a multimodal risk-reduction approach that matches the risks of illness with risks and benefits of prescribed treatment. For instance, in early-stage patients, we encourage low-risk treatments including cognitive behavior therapy, social skills training, cognitive training, family-focused psychotherapy, relaxation techniques, and neuroprotective agents such as omega-3 fatty acids. Antidepressants are used for prominent neurovegetative symptoms, although they are used with caution, as some individuals meeting criteria for prodromal psychosis, or clinical high-risk criteria, go on to develop bipolar spectrum disorders. Low-dose atypical antipsychotic treatments 
are reserved for patients closer to psychotic transition. All patients, regardless of stage of illness, are encouraged to optimize physical activity and to abstain from illicit substance use. Looking toward the future, we hope to obtain a biomarker profile (blood tests, neurocognitive, neuroimaging, and neurophysiological assessment) in each patient at the outset which, combined with clinical factors, will guide individualized treatment.

\section{Introduction}

It has become increasingly evident that at some point before the development of frank psychosis, an adolescent or young adult often begins to display subjective and objective signs of distress. Not infrequently, over the ensuing months, the young person continues to lose social and academic developmental milestones, becomes increasingly more isolated, and ultimately develops psychosis. Researchers have recognized that this period of time represents a "high-risk" state, a time to intervene and perhaps prevent psychosis from developing, or to initiate treatment immediately to reduce the $d u$ ration of untreated psychosis. The period of time when the individual has significant loss of functioning and subsyndromal psychotic symptoms has been referred to as "prodromal period," "clinical high-risk (CHR)," or "ultra-high-risk," with some differences in the definitions in the literature. The first 20 years of research in the field of CHR focused on describing the high-risk state and identifying characteristics that could predict which individuals had the highest risk of conversion to psychosis. The first North American Prodromal Longitudinal Study (NAPLS) indicated that the presence of genetic risk (first-degree relative with psychotic disorder), more severe unusual thought content, and greater social impairment predicted risk of conversion to psychosis with moderate sensitivity [1]. We have also learned that the rate of conversion is close to $30 \%$ in most cohorts and decelerates over time [1-6]. Although more is known about the risks for conversion to psychosis, the underlying mechanisms of conversion and treatment studies remain underexplored. Given the paucity of treatment studies in the field, for the purposes of our review we will use the term CHR to refer to those individuals who have been identified to be at increased risk of developing psychosis via clinical high-risk, ultrahigh-risk, or at-risk criteria. In addition, in making our recommendations, we have taken into account some studies in first-episode psychosis, which is closer to the CHR state than chronic schizophrenia.

Some special considerations in CHR populations worth mentioning at the outset include:

1. Heterogeneity and non-deterministic nature of criteria: The CHR criteria capture a wide array of young people with nonspecific symptoms and loss of functioning. As mentioned above, one-third of subjects develop psychosis. Two-thirds do not. Nonetheless, the two-thirds (nonconverters) continue to remain functionally impaired.

2. The primary outcome variable in CHR studies has been conversion to psychosis, which is different from that in studies of patients with chronic schizophrenia.

In light of these considerations, one strategy that has been proposed uses a clinical staging system that attempts to define where a given patient lies on the spectrum of a particular illness [7-9]. For instance, the use of such systems is routine in neoplastic disorders. When applied to psychosis, a clinical staging strategy could have several benefits. Broad-spectrum neuroprotective agents could be used in all stages of the illness, whereas antipsychotics may be reserved for those in advanced stages of psychosis development. In this way, treatments could be targeted to each individual based on their symptoms and place on the psychosis conversion spectrum. 


\section{Treatment}

Diet and lifestyle

- Diet and lifestyle have both direct and indirect effects on individuals in the early stages of psychosis.

- Individuals with schizophrenia have mortality rates two to three times higher than the average population, in part due to poor diet and lifestyle that are present in all stages of psychosis [10-13].

- Few studies have examined diet and lifestyle interventions in CHR subjects, although reviews $[14 \bullet \bullet, 15]$ of 26 studies of metabolic complications in FE and unmedicated patients concluded that a combination of genetic risk, unhealthy diet and lifestyle, and low

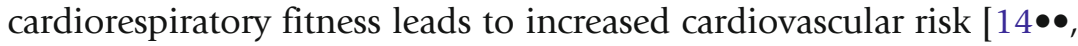
15]. First-episode individuals $(n=89)$ also reported greater health complaints, especially respiratory complaints, due to increased consumption of fast-food diets and smoking [16].

- In addition to their direct effects, poor diet and lifestyle are associated with higher levels of stress. The stress-vulnerability model of illness suggests that neurocognitive vulnerabilities (genetic trait factors), combined with environmental stressors (early-childhood stress, poor diet, poor lifestyle), trig-

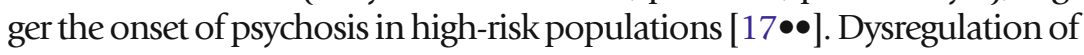
the hypothalamic-pituitary-adrenal (HPA) axis has been associated with

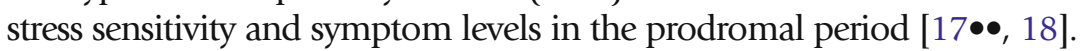
In addition, CHR populations have elevated salivary cortisol levels [19•] that are associated with increased risk of conversion to psychosis [20, 21].

- The use of illicit substances is especially relevant in CHR populations. In

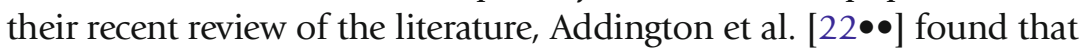
approximately $10-32 \%$ of CHR subjects had current or past use of cannabis, variable alcohol use (6-30\%), and minimal use of other substances. In particular, cannabis use has been associated with increased risk of conversion to psychosis in some studies [1,23].

Given the available evidence and emerging relationships among diet, lifestyle, and stress-vulnerability, we suggest the following in the treatment of CHR individuals:

- Close monitoring of weight and metabolic parameters to establish a risk profile based on factors such as obesity, hypertension, hyperglycemia, and diabetes

- Patient education to motivate improvements in lifestyle through the use of behavioral interventions and exercise

- Regulation of diet

- Smoking cessation 
- Addressing drug use and providing psychoeducation regarding illicit substances

- Implementation of stress-reduction programs that teach individuals to take an active role in reducing stress responses

- Cognitive behavioral therapy (discussed in detail in section on psychotherapy) approaches that help individuals to reinterpret stressful stimuli in a more adaptive way

- Family therapy (discussed in detail in section on psychotherapy) to reduce stressful familial interactions and gain support

\section{Pharmacologic treatment}

At present, pharmacologic treatment of CHR subjects is based on the critical assumption that the same treatments that reduce symptoms in patients with psychosis will be effective in individuals at CHR of developing psychosis. This is an important consideration, as the pathophysiology of the CHR brain and the chronic patient's brain may or not be the same. Few clinical trials have assessed the efficacy of psychotropic medications in treating CHR symptoms. A summary of findings from these trials is presented below.

\section{Antipsychotic medications}

Atypical antipsychotic medications have been used largely to reduce positive symptoms in CHR subjects. Trials have shown benefits from risperidone [24], olanzapine [25], amisulpride [26], and aripiprazole[27]. Overall, antipsychotic treatment in these studies reduced positive symptoms but did not have a significant impact on transition to psychosis. In addition, antipsychotic medications were associated with metabolic side effects, sometimes to a greater extent in adolescent CHR participants than older adults with SCZ (discussed above under dietary and lifestyle interventions).

In a recently completed trial of cognitive therapy+ risperidone, cognitive therapy+placebo, and supportive therapy+ placebo, McGorry et al. found no differences among the groups on their primary outcome variable, conversion to psychosis, even with 12-month follow-up [24]. They did, however, find improvements in negative symptoms and overall function in all three groups [24].

Dosage $\quad 0.5-1 \mathrm{mg}$, divided dosing initially, can be titrated up to $6 \mathrm{mg}$

Contraindications

None

Main drug interactions

Increased plasma levels when combined with CYP2D6 inhibitors and decreased levels with CYP2D6 inducers. Use caution with CNS depressants.

Main side effects somnolence, increased appetite, extrapyramidal symptoms, insomnia, vomiting, headache, coughing, constipation, orthostatic hypotension

Special points May increase prolactin levels and affect sexual function/fertility, which can be a significant concern in young adults

Cost Average $\$ 654$ per month at $8 \mathrm{mg} /$ day 
Olanzapine

Aripiprazole

Main side effects

Special points

Cost
Woods et al. conducted an 8-week randomized clinical trial with olanzapine vs. placebo in CHR subjects and found improvements in prodromal symptoms. They also noted significant weight gain (9.9 lbs. versus $0.7 \mathrm{lbs}$. in olanzapine and placebo groups, respectively). In a longer study, McGlashan et al. examined the effects of olanzapine versus placebo in CHR subjects over a 1-year period [25]. Conversion to psychosis did not meet statistical significance, but olanzapine reduced positive prodromal symptoms and was associated with significant weight gain (19.3 lbs. in olanzapine group vs. $0.7 \mathrm{lbs}$. in the placebo group) [25].

Dosage $2.5 \mathrm{mg}$ daily, can be increased to $30 \mathrm{mg}$

Contraindications None

Increased plasma levels when combined with CYP2D6 and CYP1A2 inhibitors, and decreased levels with CYP1A2 inducers. Use caution with CNS depressants.

somnolence, increased appetite and weight gain, extrapyramidal symptoms, elevated transaminases, orthostatic hypotension, risk of metabolic syndrome

Close monitoring of weight, serum glucose, and lipids

Cost: Average $\$ 546$ per month at $10 \mathrm{mg} /$ day

\section{Woods et al. conducted an open-label trial of aripiprazole in $15 \mathrm{CHR}$} subjects over 8 weeks. The authors reported improved scores on Scale of Prodromal Symptoms as early as the first week of treatment and no conversions to psychosis [27]. Treatment was accompanied by mean weight gain of 2.6 lbs. and akathisia in 8 of 13 study completers, which resolved by end of study [27].

\section{Dosage \\ Contraindications \\ Main drug interactions \\ Main side effects}

Special points

$15 \mathrm{mg}$ to $30 \mathrm{mg}$ daily

None

None

dizziness, insomnia or somnolence, extrapyramidal symptoms, nausea and vomiting, orthostatic hypotension, possible weight gain and risk of metabolic syndrome (although less frequent than other antipsychotics)

Consider carefully in individuals with comorbid anxiety; monitor weight, serum glucose, and lipids.

Cost Cost: Average $\$ 712$ per month at $15 \mathrm{mg} /$ day

\section{Antidepressants}

Antidepressant treatment has shown efficacy in a limited number of trials. It has been noted that antidepressants have lower rates of noncompliance in CHR subjects compared to antipsychotic medications, which may, in part, account for increased efficacy. In a naturalistic study of 48 CHR adolescents who were treated with antidepressants or antipsychotics, Cornblatt and colleagues found that positive symptoms improved similarly in both groups and nonadherence was a significant problem in the antipsychotic group compared to the antidepressant group [28]. 


\section{0mega-3 fatty acids (03FAs)}

The O3FAs such as eicosapentaenoic acid (EPA) and its derivative, docosahexaenoic acid (DHA), are known to exert anti-inflammatory actions [29]. In a 12-week randomized double-blind placebo-controlled trial $(\mathrm{n}=81$ CHR subjects) Amminger et al. [30] found significant reduction in conversion to psychosis in the active-treatment group. In addition, O3FAs significantly reduced positive, negative, and general symptoms and improved functioning compared to placebo. The O3FA EPA has been associated with increased levels of the antioxidants glutamine+glutamate complex, and improvement in negative symptoms [31]. EPA treatment has also been associated with increased N-acetyl-aspartate (NAA) levels, a neurotrophic metabolite, in depressed bipolar patients [32]. The effect of an EPA/DHA combination plus cognitive behavioral case management on CHR subjects is currently under investigation by McGorry et al. The NAPLS consortium is also investigating EPA/DHA effects in CHR subjects.

Given the available evidence, we suggest 1) limited use of psychotropic medications in general in the prodromal period, 2) use of omega-3 fatty acids early 3 ) starting with psychotropics with higher tolerability and lower long-term risks (antidepressants before antipsychotics), and 4) psychotropics that take individual factors into account. We recommend the use of psychotropic medications as part of a larger treatment plan that includes individual and group psychotherapy and significant dietary and lifestyle modifications. We recommend the following:

- Institute psychoeducation to promote understanding of medications, side effects, and efficacy in order to promote medication adherence.

- Consider antidepressant medications in individuals with depression with neurovegetative features. Caution is advised, as some CHR subjects may convert to bipolar spectrum illness, which could be exacerbated with antidepressant medications.

- Consider antipsychotic medications in CHR subjects with prominent positive symptoms. Use low doses of weight-neutral agents aripiprazole or risperidone - since metabolic implications in adolescents could be more prominent.

1. Cognitive behavioral herapy for psychosis (CBTp): CBT is oriented toward achieving specific goals by decreasing negative cognitions and self-schemas and increasing coping strategies. Because CBT was originally developed for mood disorders and has proven to be highly effective in anxiety disorders, it serves as a logical intervention for nonspecific emotional problems observed in CHR individuals [33]. In their recent meta-analysis, Hulton and Taylor [34] concluded that CBT significantly reduced the rate of conversion to first-episode psychosis at 6,12 , and 18-24 months following treatment in comparison to individ- 
uals who received monitoring or nonspecific supportive therapy. Moreover, the relative risk of transition to psychosis was reduced by more than $50 \%$ at every time point for individuals receiving CBT. Hafner et al. (2013) also found significantly fewer conversions at a 6-month follow-up for those CHR individuals who underwent CBT $(5.3 \%)$ in comparison to those receiving clinical management (14.8\%). A derivative of CBTp is the combination of CBT plus social skills training (SST). This combined program (CBSST) has shown efficacy in individuals with schizophrenia and may show benefit in CHR subjects [35-37].

2. Family therapy: Family psychoeducation is characterized by a set of psychotherapeutic strategies aimed at developing a collaborative relationship among the family, patient, and treatment team to stimulate recovery. There are multiple models of family therapy. These programs have in common the following features: long-term ( 9 to 24 months) treatment, delivery by a mental health professional, whole-family focus, education about schizophrenia and treatment, stress-reduction strategies, and future-oriented goal development rather than ruminating on the past [13]. A pilot study using psychoeducational multi-family group (PMFG) treatment [38] and another family-focused therapy [39] in CHR have both shown encouraging results. In FE patients, Bird et al. reviewed three trials that used family therapy as an early psychosis intervention. By the end of treatment, participants receiving family intervention therapy were less likely to relapse or be admitted to a hospital compared to those receiving standard care [40], indicating a positive effect on functioning.

3. Multi-element intervention therapy: Multi-element intervention therapy includes a combination of community outreach, case management, individual therapy, and family therapy. Multi-element treatment in early psychosis has shown multiple benefits in the domains of symptom reduction, adherence and retention in treatment, quality of life, and social functioning [13]. In the only U.S. study to date, Uzenoff et al. [41] found significant improvements in global functioning and symptoms within the first year [41].

Despite the lack of studies in CHR subjects, promising data from first-episode trials suggest benefits from psychotherapeutic modalities, and based on these results, future investigation of specific therapies aimed at CHR subjects is warranted. In addition to individual psychotherapies, multimodal treatment should also be tested, given the effects of combined treatments discussed above.

We Suggest the Following Approach to Psychotherapy in CHR Patients:

- Implementing CBT to target negative cognitions that can be part of mood, anxiety, or subsyndromal symptoms

- Implementing family therapy to bring cohesion among the affected individual, family members, and treatment team

- Considering a multimodal therapy approach that combines CBT, 


\section{Emerging treatments}

family therapy, as well as case management targeted to restore functioning

- Most psychotherapy interventions range in duration from 3 months to 1 year, with weekly visits 1-2 times a week. Therefore, establishing rapport with the affected individual and the family unit from the outset is critical.

A number of newer treatments are in varied phases of development. These include novel pharmacologic agents, psychotherapy specifically for CHR subjects, and other non-pharmacologic neurotherapeuctics.

Pharmacologic treatments

\section{Anti-inflammatory compounds (celecoxib and aspirin)}

Recent studies in early-psychosis individuals suggest that anti-inflammatory interventions may attenuate progressive brain changes [42]. For instance, the anti-inflammatory compound celecoxib, a preferential cyclooxygenase2 (COX-2) inhibitor, given in conjunction with the antipsychotic amisulpride [43] was more effective in treating negative symptoms when initiated in the early phase of schizophrenia. Aspirin (COX-1 and COX-2 inhibitor), another anti-inflammatory compound, has also shown benefit on symptoms of a subset of schizophrenia patients with relatively high anti-inflammatory cytokine production and within 10 years of onset of illness [44].

\section{Minocycline}

The broad-spectrum antibiotic minocycline is thought to have neuroprotective properties and efficacy particularly for negative symptoms of schizophrenia. It has been suggested that minocycline may play a significant role in reducing disease burden in developing countries, where infectious etiologies may contribute significantly to the schizophrenia syndrome. In one study, minocycline administered in conjunction with antipsychotic drugs improved outcomes compared to antipsychotic drugs alone in early psychosis [45].

\section{Oxytocin}

The compound oxytocin (OT) represents a novel category of small neuropeptide hormones found in the central nervous system. OT is thought to exert its effects by modulating anxiety and promoting bonding and attachment through central mechanisms. Single-dose intranasal OT administration has shown positive effects on measures of social cognition, affect recognition, and reduction in amygdala activation in response to fearful stimuli [46-49]. In the few clinical trials to date, OT has shown improvements in positive and negative symptoms, as well as improvements in short-term verbal memory. In addition to its direct pharmacologic effects, OT has been used to augment social cognition training in 
patients with SCZ, where it appears to have unique effects on acquisition of empathic accuracy.

\section{Glutamatergic neurotransmission modulators}

One particular line of drug development for schizophrenia builds on the glutamatergic theory. Excess glutamate and decreased N-methyl D-aspartate receptor (NMDAR) function is thought to precipitate glutamatergic neurotoxicity, and trials are underway of compounds that reduce glutamate availability through glutamate blockade or increased gamma-amino butyric acid (GABA). There are no studies in CHR subjects to date. Recent reports in chronic patients have reported that the selective glutamate agonist LY404039 either improved positive and negative symptoms of schizophrenia [50] or had no effect compared to placebo [51]. Increasing the availability of glycine, a naturally occurring amino acid that regulates NMDAR function, has also been hypothesized to improve symptoms of schizophrenia, and some studies have explored the effects of glycine transporter (GlyT1) inhibitors such as sarcosine (N-methylglycine), Dserine, and cycloserine in patients with schizophrenia [52, 53]. Based on positive results from studies in chronic schizophrenia, studies in CHR subjects are underway.

\section{Non-pharmacologic emerging treatments}

More recent developments in the field include non-pharmacologic strategies such as cognitive remediation, cognitive training, and neurofeedback.

\section{Cognitive remediation/cognitive training}

Cognitive remediation (CR) represents a broad set of activities and exercises whose purpose is to restore or improve cognitive functioning, which in turn is expected to improve community functioning in patients with schizophrenia. Cognitive training may be carried out using computerized programs (CogRehab software) or therapist-guided paper-and-pencil training in cognitive domains such as verbal and visual memory, language, visuomotor skills, orientation, vigilance, and processing speed. Training consists of 1- to 2-hour sessions several times a week during course of therapy. There are several variations in terms of the specifics of CR programs. Some emphasize training isolated cognitive skills, while others include vocational training, social skills training, and/or group and individual sessions. Several meta-analyses conducted to date have shown benefits that persist beyond the training period in chronic schizophrenia, irrespective of the specifics of training [54]. Two studies in CHR subjects have shown improvement in cognitive skills, specifically verbal memory [55], and lower rate of transition to psychosis [56].

Neurofeedback is a specific form of biofeedback that "feeds back" biological information regarding brain activity to a patient and asks him or her to change that activity within certain parameters. For instance, 
in some studies, a visual metaphor of pain has been used to show a patient the extent to which the anterior cingulate cortex (ACC) is activated, and the patient is then asked to reduce that activation [57]. In this way, neurofeedback is thought to train affected brain regions or circuits by optimizing voluntary control of those regions [57]. The "feedback" can be provided either in the form of brain EEG tracings or brain activation patterns seen in functional neuroimaging [57-59]. More recently, realtime functional magnetic resonance imaging (rt-fMRI) has been used in the context of chronic pain, tinnitus, depression, and schizophrenia [57-59]. After 2 weeks of training, schizophrenia patients showed brain changes consistent with learning effects and some improvement in emotion-recognition tasks [60 ${ }^{\bullet}$. It remains to be seen whether these brain changes correlate with improved function in everyday lives of patients in larger studies with clinical endpoints.

\section{Substance use disorders treatment}

At present, there are no studies addressing treatment of substance use disorders specifically in CHR individuals, using either pharmacologic or psychotherapeutic treatments. In FE populations where prevalence of cannabis and alcohol use disorders is closer to $50 \%$, Wisdom et al. [61] reviewed treatment studies with and without specialized substance use treatment. Abstinence was achieved early in the course of treatment, sometimes by 6 weeks, in half of the subjects, in both specialized and non-specialized treatments. In light of these data, the authors suggested beginning a psychoeducational program at the outset of illness and instituting specialized treatments 3-6 months later in those patients who continue to struggle with substance use [61]. A similar approach could benefit from investigation in CHR patients.

\section{Pediatric considerations}

Since the CHR period often begins during adolescence, important considerations for treatment include careful monitoring of medication side effects. Antipsychotics can lead to pronounced weight gain, which can be especially pronounced in pediatric patients. In addition, some psychotropic medications can affect sexual function and should be considered carefully.

\section{Conclusions}

At present, there are few published randomized clinical trials of treatments in CHR individuals. Investigators in the field have recognized this paucity of data, and systematic investigations are underway [62]. The primary outcome variables in these studies include conversion to psychosis, symptom reduction, and global functioning, thus rendering them especially relevant in CHR individuals. Based on available published literature, we recommend implementing strategies to 1) promote healthy lifestyle, including an open and detailed discussion of substance use and its relationship to psychosis, 2) 
increase resiliency, and 3) institute targeted multimodal treatment incorporating both pharmacologic and psychotherapeutic modalities. These include:

- Implementation of stress-reduction programs that teach individuals to take an active role in reducing stress responses

- Cognitive therapy approaches that help individuals reinterpret stressful stimuli in a more adaptive way

- Family therapy to reduce stressful familial interactions and gain support

- Combinations of psychotherapeutic modalities that potentiate the gains from each individual component and restore psychosocial functioning

- Close monitoring of patients' weight and metabolic parameters through the establishment of a risk profile based on medical factors such as obesity, hypertension, hyperglycemia, and diabetes

- Psychoeducation driven to motivate improvements in lifestyle through the use of behavioral interventions and exercise

- Smoking cessation

- Psychoeducation regarding drug use and associated risk, followed by targeted specialized treatment of substance use disorders in individuals with persistent drug use 3-6 months after initiating psychoeducation

- Regulation of diet

- Instituting psychoeducation to promote understanding of medications, side effects, and efficacy to promote medication adherence, as most CHR subjects have never been on a psychotropic medication.

- Consideration of antidepressant medications in individuals with depression with neurovegetative features. Caution is advised, as some CHR subjects may convert to bipolar spectrum illness, which could be exacerbated with antidepressants.

- Consideration of antipsychotic medications in CHR subjects in advanced prodromal stages. Use low doses of weight-neutral agents (aripiprazole or risperidone), as metabolic implications in adolescents could be more profound. Match individual preferences with side-effect profile to avoid nonadherence, which is a major limitation of antipsychotic treatment efficacy in adolescents.

\section{Compliance with Ethics Guidelines}

\section{Conflict of Interest}

Fiza Singh declares that she has no conflict of interest. Meriah DeJoseph declares that she has no conflict of interest.

Kristin S. Cadenhead declares that she has no conflict of interest.

Human and Animal Rights and Informed Consent

This article does not contain any studies with human or animal subjects performed by any of the authors. 
Papers of particular interest, published recently, have been highlighted as:

- Of importance

$\bullet \quad$ Of major importance

1. Cannon TD, Cadenhead K, Cornblatt B, Woods SW, Addington J, Walker E, et al. Prediction of psychosis in youth at high clinical risk: a multisite longitudinal study in North America. Arch Gen Psychiatry. 2008;65(1):28-37. doi:10.1001/ archgenpsychiatry.2007.3.

2. Mason O, Startup M, Halpin S, Schall U, Conrad A, Carr V. Risk factors for transition to first episode psychosis among individuals with 'at-risk mental states'. Schizophr Res. 2004;71(2-3):227-37. doi:10.1016/j.schres.2004.04.006.

3. Yung AR, Phillips LJ, Yuen HP, McGorry PD. Risk factors for psychosis in an ultra high-risk group: psychopathology and clinical features. Schizophr Res. 2004;67(2-3):131-42. doi:10.1016/S09209964(03)00192-0.

4. Yung AR, Stanford C, Cosgrave E, Killackey E, Phillips L, Nelson B, et al. Testing the ultra high risk (prodromal) criteria for the prediction of psychosis in a clinical sample of young people. Schizophr Res. 2006;84(1):57-66. doi:10.1016/j.schres.2006.03.014.

5. Yung AR, Phillips LJ, Yuen HP, Francey SM, McFarlane CA, Hallgren M, et al. Psychosis prediction: 12-month follow up of a high-risk ("prodromal") group. Schizophr Res. 2003;60(1):21-32.

6. Riecher-Rossler A, Gschwandtner U, Aston J, Borgwardt S, Drewe M, Fuhr P, et al. The Basel earlydetection-of-psychosis (FEPSY)-study-design and preliminary results. Acta Psychiatr Scand. 2007;115(2):114-25. doi:10.1111/j.16000447.2006.00854.x.

7. Kaur T, Cadenhead KS. Treatment implications of the schizophrenia prodrome. Curr Top Behav Neurosci. 2010;4:97-121.

8. Klosterkotter J, Schultze-Lutter F, Bechdolf A, Ruhrmann S. Prediction and prevention of schizophrenia: what has been achieved and where to go next? World Psychiatry. 2011;10(3):165-74.

9. Agius M, Goh C, Ulhaq S, McGorry P. The staging model in schizophrenia, and its clinical implications. Psychiatr Danub. 2010;22(2):211-20.

10. Chien WT, Leung SF, Yeung FK, Wong WK. Current approaches to treatments for schizophrenia spectrum disorders, part II: psychosocial interventions and patient-focused perspectives in psychiatric care. Neuropsychiatr Dis Treat. 2013;9:1463-81. doi:10.2147/NDT.S49263ndt-9-1463.

11. Elis O, Caponigro JM, Kring AM. Psychosocial treatments for negative symptoms in schizophrenia: cur- rent practices and future directions. Clin Psychol Rev. 2013;33(8):914-28. doi:10.1016/j.cpr.2013.07.001.

12. Clark CM. Psychosocial treatments for schizophrenia. Clin Schizophr Relat Psychoses. 2013:1-30. doi:10.3371/CSRP.CL.061213.

13. Mueser KT, Deavers F, Penn DL, Cassisi JE. Psychosocial treatments for schizophrenia. Annu Rev Clin Psychol. 2013;9:465-97. doi:10.1146/annurevclinpsy-050212-185620.

14.• Mitchell AJ, Vancampfort D, De Herdt A, Yu W, De Hert $M$. Is the prevalence of metabolic syndrome and metabolic abnormalities increased in early schizophrenia? A comparative meta-analysis of first episode, untreated and treated patients. Schizophr Bull. 2013;39(2):295-305. doi:10.1093/ schbul/sbs082.

Systematic review of literature, critical appraisal, and metaanalysis of metabolic complications in schizophrenia. Both diabetes and pre-diabetes are uncommon in early illness, smoking is elevated after diagnosis. Authors suggest prevention of cardiovascular risk accumulation rather than waiting to address it in later stages of illness when it is more difficult to treat.

15. Mitchell AJ, Vancampfort D, Sweers K, van Winkel R, $\mathrm{Yu}$ W, De Hert M. Prevalence of metabolic syndrome and metabolic abnormalities in schizophrenia and related disorders-a systematic review and metaanalysis. Schizophr Bull. 2013;39(2):306-18. doi:10.1093/schbul/sbr148.

16. Samele C, Patel M, Boydell J, Leese M, Wessely S, Murray R. Physical illness and lifestyle risk factors in people with their first presentation of psychosis. Soc Psychiatry Psychiatr Epidemiol. 2007;42(2):117-24. doi:10.1007/s00127-006-0135-2.

17.• Fisher M, Loewy R, Hardy K, Schlosser D, Vinogradov $\mathrm{S}$. Cognitive interventions targeting brain plasticity in the prodromal and early phases of schizophrenia. Annu Rev Clin Psychol. 2013;9:435-63. doi:10.1146/annurev-clinpsy-032511-143134.

This article provides a summary and detailed descriptions of cognitive psychotherapy and cognitive training in early phases of schizophrenia.

18. Corcoran C, Walker E, Huot R, Mittal V, Tessner K, Kestler $\mathrm{L}$, et al. The stress cascade and schizophrenia: etiology and onset. Schizophr Bull. 2003;29(4):671-92.

19. Walker EF, Brennan PA, Esterberg M, Brasfield J, Pearce B, Compton MT. Longitudinal changes in cortisol secretion and conversion to psychosis in atrisk youth. J Abnorm Psychol. 2010;119(2):401-8. doi:10.1037/a0018399. 
In this large multicenter prospective study, the authors examined cortisol levels at baseline and relationship between cortisol and symptom severity, as well the relationship between cortisol and conversion to psychosis. The results indicate increased baseline cortisol that correlate with increased symptom severity and indicate increased conversion risk. This study supports the emerging hypothesis regarding impaired hypothalamic-pituitary function in psychosis and has important treatment implications.

20. Walker EF, Trotman HD, Pearce BD, Addington J, Cadenhead KS, Cornblatt BA, et al. Cortisol levels and risk for psychosis: initial findings from the North American prodrome longitudinal study. Biol Psychiatry. 2013;74(6):410-7. doi:10.1016/ j.biopsych.2013.02.016.

21. Aiello G, Horowitz M, Hepgul N, Pariante CM, Mondelli V. Stress abnormalities in individuals at risk for psychosis: a review of studies in subjects with familial risk or with "at risk" mental state.

Psychoneuroendocrinology. 2012;37(10):1600-13. doi:10.1016/j.psyneuen.2012.05.003.

22.• Addington J, Case N, Saleem MM, Auther AM, Cornblatt BA, Cadenhead KS. Substance use in clinical high risk for psychosis: a review of the literature. Early Interv Psychiatry. 2013. doi:10.1111/ eip. 12100.

The authors examined the relationship between substance use disorders and conversion to psychosis. This review reveals that cannabis is a significant risk factor for at risk populations, though the prevalence of other substance use is low. In addition, there is significant variability in prevalence depending on the study reported.

23. Kristensen K, Cadenhead KS. Cannabis abuse and risk for psychosis in a prodromal sample. Psychiatry Res. 2007;151(1-2):151-4. doi:10.1016/ j.psychres.2006.10.001.

24. McGorry PD, Nelson B, Phillips LJ, Yuen HP, Francey SM, Thampi A, et al. Randomized controlled trial of interventions for young people at ultra-high risk of psychosis: twelve-month outcome. J Clin Psychiatry. 2013;74(4):349-56. doi:10.4088/JCP.12m07785.

25. McGlashan TH, Zipursky RB, Perkins D, Addington J, Miller T, Woods SW, et al. Randomized, doubleblind trial of olanzapine versus placebo in patients prodromally symptomatic for psychosis. Am J Psychiatry. 2006;163(5):790-9. doi:10.1176/ appi.ajp.163.5.790.

26. Ruhrmann S, Schultze-Lutter F, Klosterkotter J. Early detection and intervention in the initial prodromal phase of schizophrenia. Pharmacopsychiatry. 2003;36 Suppl 3:S162-7. doi:10.1055/s-2003-45125.

27. Woods SW, Tully EM, Walsh BC, Hawkins KA, Callahan JL, Cohen SJ, et al. Aripiprazole in the treatment of the psychosis prodrome: an open-label pilot study. Br J Psychiatry Suppl. 2007;51:s96-101. doi:10.1192/bjp.191.51.s96.
28. Cornblatt BA, Lencz T, Smith CW, Olsen R, Auther AM, Nakayama E, et al. Can antidepressants be used to treat the schizophrenia prodrome? Results of a prospective, naturalistic treatment study of adolescents. J Clin Psychiatry. 2007;68(4):546-57.

29. Capper EA, Marshall LA. Mammalian phospholipases $A(2)$ : mediators of inflammation, proliferation and apoptosis. Prog Lipid Res. 2001;40(3):167-97.

30. Amminger GP, Schafer MR, Papageorgiou K, Klier CM, Cotton SM, Harrigan SM, et al. Long-chain omega-3 fatty acids for indicated prevention of psychotic disorders: a randomized, placebo-controlled trial. Arch General Psychiatry. 2010;67(2):146-54. doi:10.1001/archgenpsychiatry.2009.192.

31. Berger GE, Wood SJ, Wellard RM, Proffitt TM, McConchie M, Amminger GP, et al. Ethyleicosapentaenoic acid in first-episode psychosis. A 1H-MRS study. Neuropsychopharmacol Off Publ Am Coll Neuropsychopharmacol. 2008;33(10):2467-73.

32. Frangou S, Lewis M, Wollard J, Simmons A. Preliminary in vivo evidence of increased $\mathrm{N}$-acetyl-aspartate following eicosapentanoic acid treatment in patients with bipolar disorder. J Psychopharmacol.

2007;21(4):435-9. doi:10.1177/ 0269881106067787.

33. Addington J, Epstein I, Liu L, French P, Boydell KM, Zipursky RB. A randomized controlled trial of cognitive behavioral therapy for individuals at clinical high risk of psychosis. Schizophr Res. 2011;125(1):54-61. doi:10.1016/j.schres.2010.10.015.

34. Hutton P, Taylor PJ. Cognitive behavioural therapy for psychosis prevention: a systematic review and meta-analysis. Psychol Med. 2014;44(3):449-68. doi:10.1017/S0033291713000354.

35. Granholm E, Holden J, Link PC, McQuaid JR, Jeste DV. Randomized controlled trial of cognitive behavioral social skills training for older consumers with schizophrenia: defeatist performance attitudes and functional outcome. Am J Geriatr Psychiatry. 2013;21(3):251-62. doi:10.1097/ JGP.0b013e31823e2f70.

36. Emmerson LC, Granholm E, Link PC, McQuaid JR, Jeste DV. Insight and treatment outcome with cognitive-behavioral social skills training for older people with schizophrenia. J Rehabil Res Dev. 2009;46(8):1053-8.

37. Granholm E, McQuaid JR, McClure FS, Link PC, Perivoliotis D, Gottlieb JD, et al. Randomized controlled trial of cognitive behavioral social skills training for older people with schizophrenia: 12-month followup. J Clin Psychiatry. 2007;68(5):730-7.

38. O'Brien MP, Zinberg JL, Bearden CE, Daley M, Niendam TA, Kopelowicz A, et al. Psychoeducational multi-family group treatment with adolescents at high risk for developing psychosis. Early Interv Psychiatry. 2007;1(4):325-32. doi:10.1111/j.17517893.2007.00046.x. 
39. O'Brien MP, Miklowitz DJ, Candan KA, Marshall C, Domingues I, Walsh BC, et al. A randomized trial of family focused therapy with populations at clinical high risk for psychosis: effects on interactional behavior. J Consult Clin Psychol. 2013. doi:10.1037/a0034667.

40. Bird V, Premkumar P, Kendall T, Whittington C, Mitchell J, Kuipers E. Early intervention services, cognitive-behavioural therapy and family intervention in early psychosis: systematic review. Br J Psychiatry. 2010;197(5):350-6. doi:10.1192/ bjp.bp.109.074526.

41. Uzenoff SR, Penn DL, Graham KA, Saade S, Smith BB, Perkins DO. Evaluation of a multi-element treatment center for early psychosis in the United States. Soc Psychiatry Psychiatr Epidemiol. 2012;47(10):160715. doi:10.1007/s00127-011-0467-4.

42. Meyer U. Developmental neuroinflammation and schizophrenia. Prog Neuro-Psychopharmacol Biol Psychiatry. 2011. doi:10.1016/j.pnpbp.2011.11.003.

43. Muller N, Krause D, Dehning S, Musil R, SchennachWolff R, Obermeier $M$, et al. Celecoxib treatment in an early stage of schizophrenia: results of a randomized, double-blind, placebo-controlled trial of celecoxib augmentation of amisulpride treatment. Schizophr Res. 2010;121(1-3):118-24. doi:10.1016/ j.schres.2010.04.015.

44. Laan W, Grobbee DE, Selten JP, Heijnen CJ, Kahn RS, Burger H. Adjuvant aspirin therapy reduces symptoms of schizophrenia spectrum disorders: results from a randomized, double-blind, placebo-controlled trial. J Clin Psychiatry. 2010;71(5):520-7. doi:10.4088/JCP.09m05117yel.

45. Levkovitz Y, Mendlovich S, Riwkes S, Braw Y, Levkovitch-Verbin H, Gal G, et al. A double-blind, randomized study of minocycline for the treatment of negative and cognitive symptoms in early-phase schizophrenia. J Clin Psychiatry. 2010;71(2):138-49. doi:10.4088/JCP.08m04666yel.

46. Feifel D, Shilling PD, Belcher AM. The effects of oxytocin and its analog, carbetocin, on genetic deficits in sensorimotor gating. Eur Neuropsychopharmacol J Eur Coll Neuropsychopharmacol. 2012;22(5):374-8. doi:10.1016/j.euroneuro.2011.09.004.

47. Pedersen CA, Gibson CM, Rau SW, Salimi K, Smedley KL, Casey RL, et al. Intranasal oxytocin reduces psychotic symptoms and improves theory of mind and social perception in schizophrenia. Schizophr Res. 2011;132(1):50-3. doi:10.1016/ j.schres.2011.07.027.

48. Averbeck BB, Bobin T, Evans S, Shergill SS. Emotion recognition and oxytocin in patients with schizophrenia. Psychol Med. 2011;42(2):1-8. doi:10.1017/ S0033291711001413.

49. Feifel D, Macdonald K, Nguyen A, Cobb P, Warlan H, Galangue B, et al. Adjunctive intranasal oxytocin reduces symptoms in schizophrenia patients. Biol
Psychiatry. 2010;68(7):678-80. doi:10.1016/ j.biopsych.2010.04.039.

50. Patil ST, Zhang L, Martenyi F, Lowe SL, Jackson KA, Andreev BV, et al. Activation of mGlu2/3 receptors as a new approach to treat schizophrenia: a randomized Phase 2 clinical trial. Nat Med. 2007;13(9):1102-7. doi:10.1038/nm1632.

51. Kinon BJ, Zhang L, Millen BA, Osuntokun OO, Williams JE, Kollack-Walker S, et al. A multicenter, inpatient, phase 2, double-blind, placebo-controlled dose-ranging study of LY2140023 monohydrate in patients with DSM-IV schizophrenia. J Clin Psychopharmacol. 2011;31(3):349-55. doi:10.1097/ JCP.0b013e318218dcd5.

52. Sacchi S, Rosini E, Pollegioni L, Molla G. D-amino acid oxidase inhibitors as a novel class of drugs for schizophrenia therapy. Curr Pharm Des. 2013;19(14):2499-511.

53. Javitt DC. Glycine transport inhibitors in the treatment of schizophrenia. Handb Exp Pharmacol. 2012;213:36799. doi:10.1007/978-3-642-25758-2_12.

54. Wykes T, Steel C, Everitt B, Tarrier N. Cognitive behavior therapy for schizophrenia: effect sizes, clinical models, and methodological rigor. Schizophr. Bukk. 2008;34(3):523-37.

55. Adcock RA, Dale C, Fisher M, Aldebot S, Genevsky A, Simpson GV, Nagarajan S, Vinogradov S. When topdown meets bottom-up: auditory training enhances verbal memory in schizophrenia. Schizophr. Bull. 2009;35(6):1132-41. doi:10.1093/schbul/sbp068.

56. Bechdolf A, Wagner $M$, Ruhrmann S, Harrigan $S$, Putzfeld V, et al. Preventing progression to first-episode psychosis in early initial prodromal states. Br. J. Psychiatry 2012;200(1):22-9.

57. Sulzer J, Haller S, Scharnowski F, Weiskopf N, Birbaumer N, Blefari ML, et al. Real-time fMRI neurofeedback: progress and challenges. Neuroimage. 2013;76:386-99. doi:10.1016/ j.neuroimage.2013.03.033.

58. Birbaumer N, Ruiz S, Sitaram R. Learned regulation of brain metabolism. Trends Cogn Sci. 2013;17(6):295-302. doi:10.1016/ j.tics.2013.04.009.

59. Weiskopf N. Real-time fMRI and its application to neurofeedback. Neuroimage. 2012;62(2):682-92. doi:10.1016/j.neuroimage.2011.10.009.

60. Ruiz S, Lee S, Soekadar SR, Caria A, Veit R, Kircher T, et al. Acquired self-control of insula cortex modulates emotion recognition and brain network connectivity in schizophrenia. Hum Brain Mapp. 2013;34(1):200-12. doi:10.1002/hbm.21427.

The authors used a real-time functional neuroimaging paradigm to train 9 schizophrenia patients to reduce hemodymanic response in their insular cortices. In this 2week training paradigm, results indicated a learning response at the level of brain function and increased accuracy in emotion recognition. 
61. Wisdom JP, Manuel JI, Drake RE. Substance use disorder among people with first-episode psychosis: a systematic review of course and treatment. Psychiatr Serv. 2011;62(9):1007-12. doi:10.1176/ appi.ps.62.9.1007.

62.• Correll CU, Hauser M, Auther AM, Cornblatt BA. Research in people with the psychosis risk syndrome: a review of the current evidence and future directions. J Child Psychol Psychiatry. 2010;51(4):390431.

This article provides a historical perspective on the study of prodromal schizophrenia and a summary of major research findings. Also includes a list of ongoing studies in clinically high risk populations. 